\title{
Localized Discrete Laplace-Beltrami Operator Over Triangular Mesh
}

\author{
Xinge $\mathrm{Li}^{\mathrm{a}}$, Guoliang $\mathrm{Xu}^{\mathrm{a}, *}$, Yongjie Jessica Zhang ${ }^{\mathrm{b}}$ \\ ${ }^{a}$ LSEC, ICMSEC, Academy of Mathematics and Systems Science, Chinese Academy of \\ Sciences, Beijing, China \\ ${ }^{b}$ Computational Biomodeling Laboratory, Department of Mechanical Engineering, Carnegie \\ Mellon University, Pittsburgh, USA
}

\begin{abstract}
The Laplace-Beltrami operator is the foundation of describing geometric partial differential equations, and it also plays an important role in the fields of computational geometry, computer graphics and image processing, such as surface parameterization, shape analysis, matching and interpolation. However, constructing the discretized Laplace-Beltrami operator with convergent property has been an open problem. In this paper we propose a new discretization scheme of the Laplace-Beltrami operator over triangulated surfaces. We prove that our discretization of the Laplace-Beltrami operator converges to the Laplace-Beltrami operator at every point of an arbitrary smooth surface as the size of the triangular mesh over the surface tends to zero. Numerical experiments are conducted, which support the theoretical analysis.
\end{abstract}

Keywords: Laplace-Beltrami operator, Surface triangulation, Discretization, Convergence

\section{Introduction}

The Laplace-Beltrami operator (LBO) is a generalization of the Laplace operator from flat spaces to manifolds. It plays a central role in many areas, such as image processing [1, 2, 3, 4, surface processing [5, 6, 7, 8, 9, 10, and the

\footnotetext{
* Corresponding author

Email address: xuguo@lsec.cc.ac.cn (Guoliang Xu)
}

Preprint submitted to Elsevier

September 1, 2015 
study of geometric partial differential equations (PDEs) [1, 11, 3, 12. In these applications, the objective surfaces to be processed are usually represented as discrete meshes. Hence, it is necessary in practice to discretize the LBO.

The classical Laplace operator on flat spaces is generally approximated by a finite difference method. However, due to the complexity and diversity of the discretized manifold surfaces, the discretization of the LBO is much more complicated than the Laplacian over flat spaces. In the literature, several discretizations have been proposed over triangular meshes [13, 14, 7, 11, 15, 16]. Most of them are variants of the cotangent scheme [13, which is a form of the finite element method, applied to the Laplace-Beltrami operator on a surface. Given a surface with a fine mesh, we expect the discretization computed from such a mesh provides an accurate representation of the underlying surface Laplacian. However, a detailed theoretical analysis of existing discretizations in [15. shows that while point-wise convergence can be established for special classes of meshes, such as certain meshes with valence-6, or for linear functions over a sphere in $\mathbf{R}^{3}$, none of these methods is convergent for surface meshes in general. Wardetzky [17 also provided an overview over convergence properties of weak versions of the LBO for embedded polyhedral surfaces from a functional-analytic viewpoint.

For the quadrilateral or even general polygonal meshes there are far fewer results. Liu et al. 18, described a discretization of the LBO on quadrilateral meshes based on a bilinear interpolation of the quadrilateral. They achieved consistency under some special, but not too restrictive conditions. Xiong et al. [19] obtained a discrete LBO on quadrilateral meshes by averaging the cotangent scheme over all possible triangulations of the given mesh, and Alexa et al. [20] extended the cotangent scheme to the case of general polygonal meshes. Recently, Carl 21] derived a Laplace operator on semi-discrete surface, which is represented by a mapping into $\mathbf{R}^{3}$ possessing one discrete and one continuous variable. He proved consistency of the semi-discrete Laplacian, meaning that it converges point-wise to the Laplace-Beltrami operator when the semidiscrete surface converges to a smooth one, and also showed convergence of the 
discretization of LBO on quadrilateral meshes defined in [20].

In 22], the authors introduced a point-wise convergent discretization of the LBO on the triangular mesh, called the mesh Laplacian. The construction is based on a discretization of the heat kernel defined on the flat space $\mathbf{R}^{2}$. The convergent properties have been generalized by Belkin et al. [23] to point clouds in $\mathbf{R}^{d}$, and they constructed the PCD Laplacian. Sun et al. 24] applied the mesh Laplacian to the computation of the heat kernel signature, which is obtained by restricting the heat kernel to the time domain, and derived a concise and provably informative multi-scale point signature based on the heat diffusion process. A notable recent work 25] built a new point-wisely symmetrizable discrete LBO over the point-sampled surface which is also based on the same discretization of the heat kernel. The authors also indicated that their discretization can provide orthogonal bases for further spectral geometric analysis and processing tasks. In [26], the authors proposed a principle for constructing strongly consistent discrete LBO based on the cotangent weights.

In this paper, we present a new discretization scheme that guarantees convergence at each point, which is $L_{\infty}$ convergence, and it is applicable to arbitrary triangulated surfaces. We summarize our main contributions as follows:

1. Our discretization scheme is based on a discretization of the heat kernel defined on the curved surface embedded in $\mathbf{R}^{3}$. It is much more accurate than the scheme based on the discretization of the heat kernel defined on the plane, especially for waved surfaces with insufficient dense triangulations;

2. Our discretization scheme is applicable to arbitrary triangulated surfaces without the limitation on the quality of the triangular mesh. We study how the surface and its triangulation influence the integral approximation over a surface;

3. Combining the integral approximation results with the idea of approximating the heat flow on a triangular mesh, we present a local and adaptive algorithm for approximating the LBO on an arbitrary surface with a 
point-wise convergence guarantee for triangular meshes;

4. We propose a method to estimate the parameters involved in the algorithm adaptively; and

5. We provide experimental results showing that our method outperforms other discretization schemes in terms of $L_{\infty}$ convergence.

The rest of the paper is organized as follows. In Section 2, we introduce the LBO and its approximation. In Section 3, we describe a quantitative measure of how well a triangular mesh approximates the underlying surface. In section 4, we present and analyze our method for approximating the LBO with a pointwise convergence guarantee for arbitrary triangulated surfaces. Some numerical results are presented in Section 5. Section 6 concludes the paper. Some proofs of the theoretical results are presented in the appendix.

\section{Laplace-Beltrami operator and its approximation based on the heat kernel}

In this paper, we consider a smooth compact (oriented) surface $\mathcal{S} \subset \mathbf{R}^{3}$ without boundary. Let $\mathbf{x}\left(u^{1}, u^{2}\right)=\left[x\left(u^{1}, u^{2}\right), y\left(u^{1}, u^{2}\right), z\left(u^{1}, u^{2}\right)\right]^{\mathrm{T}}$ be the parametric representation of $\mathcal{S}$, and $g_{\alpha \beta}=\left\langle\mathbf{x}_{u^{\alpha}}, \mathbf{x}_{u^{\beta}}\right\rangle(\alpha, \beta=1,2)$ be the coefficients of the first fundamental form of $\mathcal{S}$ with $\mathbf{x}_{u^{\alpha}}=\frac{\partial \mathbf{x}}{\partial u^{\alpha}}$. Set $g=\operatorname{det}\left(g_{\alpha \beta}\right),\left[g^{\alpha \beta}\right]=\left[g_{\alpha \beta}\right]^{-1}$. Let $f$ be a $C^{1}$ smooth function on $\mathcal{S}$, i.e., $f \in C^{1}(\mathcal{S})$, the tangential gradient operator $\nabla_{\mathcal{S}}$ acting on $f$ is given by

$$
\nabla_{\mathcal{S}} f=\left[\mathbf{x}_{u^{1}}, \mathbf{x}_{u^{2}}\right]\left[g^{\alpha \beta}\right]\left[f_{u^{1}}, f_{u^{2}}\right]^{\mathrm{T}} \in \mathbf{R}^{3},
$$

where $f_{u^{\alpha}}=\frac{\partial f}{\partial u^{\alpha}}$. Let $\mathbf{v}$ be a $C^{1}$ smooth vector field on surface $\mathcal{S}$, we define the tangential divergence operator $\operatorname{div}_{\mathcal{S}}$ acting on $\mathbf{v}$ by

$$
\operatorname{div}_{\mathcal{S}}(\mathbf{v})=\frac{1}{\sqrt{g}}\left[\frac{\partial}{\partial u^{1}}, \frac{\partial}{\partial u^{2}}\right]\left[\sqrt{g}\left[g^{\alpha \beta}\right]\left[\mathbf{x}_{u^{1}}, \mathbf{x}_{u^{2}}\right]^{\mathrm{T}} \mathbf{v}\right] .
$$


Let $f \in C^{2}(\mathcal{S})$, then $\nabla_{\mathcal{S}} f$ is a $C^{1}$ smooth vector field on $\mathcal{S}$, and the LaplaceBeltrami operator $\Delta_{\mathcal{S}}$ applying to $f$ is defined as

$$
\begin{aligned}
\Delta_{\mathcal{S}} f & =\operatorname{div}_{\mathcal{S}}\left(\nabla_{\mathcal{S}} f\right) \\
& =\frac{1}{\sqrt{g}}\left[\frac{\partial}{\partial u^{1}}, \frac{\partial}{\partial u^{2}}\right]\left[\sqrt{g}\left[g^{\alpha \beta}\right]\left[f_{u^{1}}, f_{u^{2}}\right]^{\mathrm{T}}\right] .
\end{aligned}
$$

Let $\mathbf{x}$ be a surface point on $\mathcal{S}$, we have [27]

$$
\Delta_{\mathcal{S}} \mathbf{x}=2 \mathbf{H}(\mathbf{x}) \in \mathbf{R}^{3}
$$

where $\mathbf{H}(\mathbf{x})$ is the mean curvature normal at $\mathbf{x}$, and $\mathbf{H}(\mathbf{x}) /\|\mathbf{H}(\mathbf{x})\|$ is the unit surface normal.

Now we consider the approximation of $\Delta_{\mathcal{S}} f$. It is well known that the solution $u(\mathbf{x}, t)$ of the heat equation on surface $\mathcal{S}$

$$
\left\{\begin{array}{l}
\Delta_{\mathcal{S}} u(\mathbf{x}, t)=\frac{\partial u}{\partial t}(\mathbf{x}, t), \\
u(\mathbf{x}, 0)=f(\mathbf{x}),
\end{array}\right.
$$

can be written as

$$
u(\mathbf{x}, t)=\int_{\mathcal{S}} H_{\mathcal{S}}^{t}(\mathbf{x}, \mathbf{y}) f(\mathbf{y}) d \nu(\mathbf{y})
$$

where $\nu$ denotes the area of a surface element, and $H_{\mathcal{S}}^{t}(\mathbf{x}, \mathbf{y})$ is the heat kernel of the surface $\mathcal{S}$, i.e., the measure of how much heat propagates from $\mathbf{y}$ to $\mathbf{x}$ in time $t$. Thus the heat equation can be rewritten as follows:

$$
\Delta_{\mathcal{S}} u(\mathbf{x}, t)=\frac{\partial}{\partial t} \int_{\mathcal{S}} H_{\mathcal{S}}^{t}(\mathbf{x}, \mathbf{y}) f(\mathbf{y}) d \nu(\mathbf{y}) .
$$

Recalling that $u(\mathbf{x}, 0)=f(\mathbf{x})$ and that

$$
\int_{\mathcal{S}} H_{\mathcal{S}}^{t}(\mathbf{x}, \mathbf{y}) d \nu(\mathbf{y})=1
$$

we obtain

$$
\begin{aligned}
\Delta_{\mathcal{S}} f(\mathbf{x}) & =\left.\frac{\partial}{\partial t} \int_{\mathcal{S}} H_{\mathcal{S}}^{t}(\mathbf{x}, \mathbf{y}) f(\mathbf{y}) d \nu(\mathbf{y})\right|_{t=0} \\
& =\lim _{t \rightarrow 0} \frac{1}{t}\left(\int_{\mathcal{S}} H_{\mathcal{S}}^{t}(\mathbf{x}, \mathbf{y}) f(\mathbf{y}) d \nu(\mathbf{y})-u(\mathbf{x}, 0)\right) \\
& =\lim _{t \rightarrow 0} \frac{1}{t}\left(\int_{\mathcal{S}} H_{\mathcal{S}}^{t}(\mathbf{x}, \mathbf{y}) f(\mathbf{y}) d \nu(\mathbf{y})-f(\mathbf{x})\right) \\
& =\lim _{t \rightarrow 0} \frac{1}{t} \int_{\mathcal{S}} H_{\mathcal{S}}^{t}(\mathbf{x}, \mathbf{y})(f(\mathbf{y})-f(\mathbf{x})) d \nu(\mathbf{y}) .
\end{aligned}
$$


However, we do not know the exact form of the heat kernel $H_{\mathcal{S}}^{t}(\mathbf{x}, \mathbf{y})$ for an arbitrary manifold with some rare exceptions, and all we can utilize is the asymptotic form. For example,

$$
H_{\mathcal{S}}^{t}(\mathbf{x}, \mathbf{y}) \approx \frac{1}{4 \pi t} e^{-\frac{d_{\mathcal{S}}(\mathbf{x}, \mathbf{y})^{2}}{4 t}},
$$

where $d_{\mathcal{S}}(\mathbf{x}, \mathbf{y})$ denotes the geodesic distance between two points $\mathbf{x}$ and $\mathbf{y}$ on surface $\mathcal{S}$. Then, given a point $\mathbf{x} \in \mathcal{S}$ and a function $f: \mathcal{S} \rightarrow \mathbf{R}$, we obtain the approximation of the $\mathrm{LBO}$, denoted as $\mathrm{F}_{\mathcal{S}}^{t}$ :

$$
\mathrm{F}_{\mathcal{S}}^{t} f(\mathbf{x})=\frac{1}{4 \pi t^{2}} \int_{\mathcal{S}} e^{-\frac{d_{\mathcal{S}}(\mathbf{x}, \mathbf{y})^{2}}{4 t}}(f(\mathbf{y})-f(\mathbf{x})) d \nu(\mathbf{y}),
$$

where the parameter $t$ is a positive quantity, which intuitively reflects the size of the neighbourhood considered at the point $\mathbf{x}$. Through the above discussion, we obtain the following theorem.

Theorem 1. For a given function $f \in C^{2}(\mathcal{S})$, we have

$$
\lim _{t \rightarrow 0}\left\|F_{\mathcal{S}}^{t} f-\Delta_{\mathcal{S}} f\right\|_{\infty}=0
$$

Remark 1. In 22], the authors selected the heat kernel as

$$
H_{\mathcal{S}}^{t}(\mathbf{x}, \mathbf{y}) \approx \frac{1}{4 \pi t} e^{-\frac{\|\mathbf{x}-\mathbf{y}\|^{2}}{4 t}},
$$

where $\|\mathbf{x}-\mathbf{y}\|$ denotes the euclidean distance between two points $\mathbf{x}$ and $\mathbf{y}$ on surface $\mathcal{S}$. But we believe that the geodesic distance is a better choice, especially for the steep surfaces. Numerical experiments in Section 5 will illustrate our statement.

From (5), we can observe that $\mathrm{F}_{\mathcal{S}}^{t}$ is global in the sense that computing the Laplacian of a function at some points requires the integral over the whole surface. Now we localize the approximation $\mathrm{F}_{\mathcal{S}}^{t}$. Let $\mathcal{S}_{r}(\mathbf{x})=\left\{\mathbf{y} \in \mathcal{S}: d_{\mathcal{S}}(\mathbf{x}, \mathbf{y}) \leq\right.$ $r\} \subset \mathcal{S}$, we approximate $\mathrm{F}_{\mathcal{S}}^{t}$ by

$$
\mathrm{F}_{\mathcal{S}_{r}}^{t} f(\mathbf{x})=\frac{1}{4 \pi t^{2}} \int_{\mathbf{y} \in \mathcal{S}_{r}(\mathbf{x})} e^{-\frac{d_{\mathcal{S}}(\mathbf{x}, \mathbf{y})^{2}}{4 t}}(f(\mathbf{y})-f(\mathbf{x})) d \nu(\mathbf{y}),
$$

and we obtain another theorem. 
Theorem 2. For a given function $f \in C^{2}(\mathcal{S})$ and a given $r>0$, we have

$$
\lim _{t \rightarrow 0}\left\|F_{\mathcal{S}_{r}}^{t} f-\Delta_{\mathcal{S}} f\right\|_{\infty}=0
$$

Proof. For any point $\mathbf{x} \in \mathcal{S}$, we have

$$
\left|\mathrm{F}_{\mathcal{S}_{r}}^{t} f(\mathbf{x})-\Delta_{\mathcal{S}} f(\mathbf{x})\right| \leq\left|\mathrm{F}_{\mathcal{S}_{r}}^{t} f(\mathbf{x})-\mathrm{F}_{\mathcal{S}}^{t} f(\mathbf{x})\right|+\left|\mathrm{F}_{\mathcal{S}}^{t} f(\mathbf{x})-\Delta_{\mathcal{S}} f(\mathbf{x})\right|
$$

For the first term on the right hand side, we have

$$
\begin{aligned}
\left|\mathrm{F}_{\mathcal{S}_{r}}^{t} f(\mathbf{x})-\mathrm{F}_{\mathcal{S}}^{t} f(\mathbf{x})\right| & =\left|\frac{1}{4 \pi t^{2}} \int_{\mathbf{y} \in \mathcal{S} \backslash \mathcal{S}_{r}(\mathbf{x})} e^{-\frac{d_{\mathcal{S}}(\mathbf{x}, \mathbf{y})^{2}}{4 t}}(f(\mathbf{y})-f(\mathbf{x})) d \nu(\mathbf{y})\right| \\
& \leq \frac{1}{4 \pi t^{2}} \int_{\mathbf{y} \in \mathcal{S} \backslash \mathcal{S}_{r}(\mathbf{x})} e^{-\frac{d_{\mathcal{S}}(\mathbf{x}, \mathbf{y})^{2}}{4 t}}|f(\mathbf{y})-f(\mathbf{x})| d \nu(\mathbf{y}) \\
& \leq \frac{2\|f\|_{\infty} A(\mathcal{S})}{4 \pi t^{2}} e^{-\frac{r^{2}}{4 t}}
\end{aligned}
$$

where $A(\mathcal{S})$ denotes the area of the surface $\mathcal{S}$. Using L'Hospital's rule, we can obtain

$$
\begin{aligned}
\lim _{t \rightarrow 0} \frac{1}{t^{2}} e^{-\frac{r^{2}}{4 t}} & =\lim _{t \rightarrow 0} \frac{t^{-2}}{e^{\frac{r^{2}}{4 t}}} \\
& =\lim _{t \rightarrow 0} \frac{-2 t^{-3}}{-\frac{r^{2} t^{-2}}{4} e^{\frac{r^{2}}{4 t}}}=\lim _{t \rightarrow 0} \frac{8 t^{-1}}{r^{2} e^{\frac{r^{2}}{4 t}}} \\
& =\lim _{t \rightarrow 0} \frac{-8 t^{-2}}{-\frac{r^{4} t^{-2}}{4} e^{\frac{r^{2}}{4 t}}}=\lim _{t \rightarrow 0} \frac{32}{r^{4} e^{\frac{r^{2}}{4 t}}}=0 .
\end{aligned}
$$

Combining it with Eqn (9), we have

$$
\lim _{t \rightarrow 0}\left|\mathrm{~F}_{\mathcal{S}_{r}}^{t} f(\mathbf{x})-\mathrm{F}_{\mathcal{S}}^{t} f(\mathbf{x})\right|=0 .
$$

On the other hand, we obtain the limit

$$
\lim _{t \rightarrow 0}\left|F_{\mathcal{S}}^{t} f(\mathbf{x})-\Delta_{\mathcal{S}} f(\mathbf{x})\right|=0
$$

by Theorem 1. Hence, by taking the limit, we obtain $\lim _{t \rightarrow 0} \mathrm{~F}_{\mathcal{S}_{r}}^{t} f(\mathbf{x})=\Delta_{\mathcal{S}} f(\mathbf{x})$, which proves the theorem.

Theorems 1 and 2 state that these two approximate operators $\mathrm{F}_{\mathcal{S}}^{t}$ and $\mathrm{F}_{\mathcal{S}_{r}}^{t}$ converge to the LBO at every point on the surface. 


\section{Triangulation of the surface and measurement of the triangulation}

Let $K$ be a triangular mesh in $\mathbf{R}^{3}$. We now propose a quantitative measure of how well a triangular mesh approximates the underlying surface. We first cite a lemma from 28 .

Lemma 3. Let $\mathcal{S}$ be a smooth compact (oriented) surface in $\mathbf{R}^{3}$, there exists an open set $U_{\mathcal{S}}$ of $\mathbf{R}^{3}$ containing $\mathcal{S}$ and a continuous map $\xi$ from $U_{\mathcal{S}}$ onto $\mathcal{S}$ satisfying the following property: if $\boldsymbol{p}$ belongs to $U_{\mathcal{S}}$, then there exists a unique point $\xi(\boldsymbol{p})$ realizing the distance from $\boldsymbol{p}$ to $\mathcal{S}$ ( $\xi$ is the orthogonal projection onto $\mathcal{S})$.

A proof of this lemma can be found in [28. We shall also need the notion of the reach of a surface, introduced in $[28$.

Definition 1. The reach of a smooth compact surface $\mathcal{S}$ is the largest $\rho>0$ for which $\xi$ is defined on the (open) tubular neighbourhood $U_{\rho}(\mathcal{S})$ of radius $\rho$ of $\mathcal{S}$.

Definition 2. We say that a triangular mesh $K$ is closely inscribed in a smooth surface $\mathcal{S}$ if

(1) all the vertices of $K$ lie on $\mathcal{S}$;

(2) $K$ lies in $U_{\rho}(\mathcal{S})$ where $\rho$ is the reach of $\mathcal{S}$; and

(3) the restriction of $\xi$ to $K$, i.e., $\xi: K \rightarrow \mathcal{S}$, is bijective.

Here, we assume that the triangular mesh $K$ is closely inscribed in $\mathcal{S}$. We denote the set of triangles of $K$ as $\mathcal{T}_{K}$ and denote a generic triangle of $K$ as $\triangle$. $\eta(\triangle)$ represents the length of the longest edge of $\triangle$. Let $V(\triangle)$ denote the set of vertices of $\triangle$, and then we have

$$
\operatorname{rig}(\triangle)=\sup _{\mathbf{p} \in V(\triangle)}\left|\sin \left(\theta_{\mathbf{p}}\right)\right|,
$$

where $\theta_{\mathbf{p}}$ is the angle at vertex $\mathbf{p}$ of $\triangle$, denoting the rightness of a triangle $\triangle$ (Figure 1), and we have $0<\operatorname{rig}(\triangle) \leq 1$. Globally, we denote

$$
\eta(K)=\sup _{\triangle \in \mathcal{T}_{K}} \eta(\triangle)
$$


as the height of $K$, and denote

$$
\operatorname{rig}(K)=\min _{\triangle \in \mathcal{T}_{K}} \operatorname{rig}(\triangle)
$$

as the rightness of $K$.

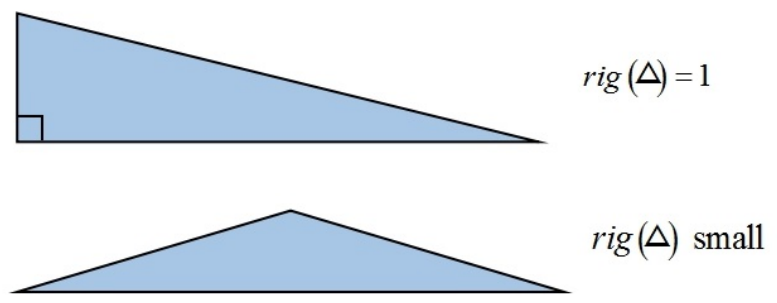

Figure 1: Rightness of a triangle $\triangle$.

Definition 3. We say that $K$ is a sufficiently fine triangular mesh $K_{\varepsilon, \delta}$ of $\mathcal{S}$ if there exist two positive constants $\varepsilon$ and $\delta$, such that

$$
\eta(K) \leq \varepsilon \rho, \quad \operatorname{rig}(K) \geq \delta
$$

We will later need the following results on the relations between a smooth surface $\mathcal{S}$ and an approximating triangular mesh $K_{\varepsilon, \delta}$ of $\mathcal{S}$. Let $\mathbf{n}_{\mathbf{p}}$ denote the unit normal of $\mathcal{S}$ at the point $\mathbf{p}$ and $\mathbf{n}_{\triangle}$ the normal of the triangle $\triangle$. The following lemma from [29] compares the behaviour of the normal vector field of a smooth surface $\mathcal{S}$ with the normal of each face of a triangulation $K_{\varepsilon, \delta}$.

Lemma 4. Let $K_{\varepsilon, \delta}$ be an approximating triangular mesh of $\mathcal{S}$, then we have

$$
\sin \angle\left(\boldsymbol{n}_{\triangle}, \boldsymbol{n}_{\boldsymbol{p}}\right) \leq\left(\frac{4}{\delta}+2\right) \varepsilon
$$

for any triangle $\triangle \in \mathcal{T}_{K}$ and any point $\boldsymbol{p} \in V(\triangle)$.

For any point $\mathbf{p} \in \mathbf{R}^{3}$, let $d(\mathbf{p}, S)$ denote the shortest distance from $\mathbf{p}$ to any point in the set $S \subset \mathbf{R}^{3}$, and we have the following lemma.

Lemma 5. Let $K_{\varepsilon, \delta}$ be an approximating triangular mesh of $\mathcal{S}$, then we have

(i) For any point $\boldsymbol{p} \in K, d(\boldsymbol{p}, \mathcal{S}) \leq\left(\frac{4}{\delta}+3\right) \varepsilon^{2} \rho$; and 
(ii) For any point $\boldsymbol{x} \in \mathcal{S}, d(\boldsymbol{x}, K) \leq\left(\frac{4}{\delta}+3\right) \varepsilon^{2} \rho$.

Remark 2. Results like this lemma have been proposed in Lemma 3.3 in [22].

We put the proof of this lemma into the Appendix of the paper.

\section{Our discretization of Laplace-Beltrami operator and its conver- gence results}

In this section, we present our local algorithm for approximating the LBO on an arbitrary surface by constructing the discretized version of the integral 7).

First, we construct a discretized version of the integral $\int_{\mathcal{S}} g(\mathbf{x}) d \nu(\mathbf{x})$, where $g: \mathcal{S} \rightarrow \mathbf{R}$ is a function defined on $\mathcal{S}$, by setting

$$
\mathrm{I}_{K} g=\sum_{\triangle \in K} \frac{A(\triangle)}{3} \sum_{\mathbf{p} \in V(\triangle)} g(\mathbf{p})
$$

where $A(\triangle)$ denotes the area of the triangle $\triangle$. That is, each triangle of the mesh contributes to $\mathrm{I}_{K} g$ the amount of its area multiplied by the average value of its vertices.

Lemma 5 implies that the triangular mesh $K$ is close to the underlying surface $\mathcal{S}$ both geometrically and topologically. Intuitively, quantities defined on $\mathcal{S}$ are closely related to their analogs defined on $K$. Indeed, consider an arbitrary but fixed triangle $\triangle \in \mathcal{T}_{K}$, note that although the map $\xi$ is not differentiable on the entire domain $K$, it is differentiable for the interior of $\triangle$. We now present a result on bounding the Jacobian of the map $\xi$.

Lemma 6. Let $K_{\varepsilon, \delta}$ be an approximating triangular mesh of $\mathcal{S}$ with $\varepsilon<1 / 10$ and $\delta \geq 4 / 37$. Given any triangle $\triangle \in \mathcal{T}_{K}$, for any point $\boldsymbol{p}$ in the interior of $\triangle, \xi$ is differentiable at $\boldsymbol{p}$ and we have

$$
|J(\boldsymbol{p})| \leq \frac{1}{1-\left(\frac{4}{\delta}+3\right) \varepsilon^{2}},
$$

where $J(\boldsymbol{p})$ is the Jacobian of the map $\xi$ at the point $\boldsymbol{p}$. In particular,

$$
\left|\frac{A(\mathcal{S})}{A\left(K_{\varepsilon, \delta}\right)}\right| \leq \frac{1}{1-\left(\frac{4}{\delta}+3\right) \varepsilon^{2}},
$$


where $A(\mathcal{S})$ and $A\left(K_{\varepsilon, \delta}\right)$ denote the areas of $\mathcal{S}$ and $K_{\varepsilon, \delta}$ respectively.

Proof. By Lemma 1 in [30, we have

$$
|J(\mathbf{p})| \leq \frac{1}{1-\|\xi(\mathbf{p})-\mathbf{p}\| \lambda_{\xi(\mathbf{p})}}
$$

where $\lambda_{\xi(\mathbf{p})}$ is the maximal curvature of $\mathcal{S}$ at $\xi(\mathbf{p})$. Combining 12 with

$$
\lambda_{\xi(\mathbf{p})} \leq \frac{1}{\rho}
$$

and Lemma 5 (i) above, we obtain

$$
|J(\mathbf{p})| \leq \frac{1}{1-\left(\frac{4}{\delta}+3\right) \varepsilon^{2}}
$$

which proves the lemma.

The following lemma from 22 bounds the geodesic distance $d_{\mathcal{S}}(\mathbf{p}, \mathbf{q})$ in terms of the Euclidean distance $\|\mathbf{p}-\mathbf{q}\|$ between points $\mathbf{p}$ and $\mathbf{q}$.

Lemma 7. Given two points $\boldsymbol{p}, \boldsymbol{q} \in \mathcal{S}$ and letting $d=\|\boldsymbol{p}-\boldsymbol{q}\|<\rho / 2$, we have $d \leq d_{\mathcal{S}}(\boldsymbol{p}, \boldsymbol{q}) \leq d+\frac{4 d^{3}}{3 \rho^{2}}$.

The following theorem shows the relation between $\int_{\mathcal{S}} g(\mathbf{x}) d \nu(\mathbf{x})$ and $\mathrm{I}_{K} g$ in terms of the parameters of the surface and the mesh.

Theorem 8. Given a Lipschitz function $g: \mathcal{S} \rightarrow \mathbf{R}$, let $L=\operatorname{Lip}(g)$ be the Lipschitz constant of function $g$, i.e., $|g(\boldsymbol{x})-g(\boldsymbol{y})| \leq \operatorname{Lip}(g) d_{\mathcal{S}}(\boldsymbol{x}, \boldsymbol{y})$. Setting $\|g\|_{\infty}=\sup _{x \in \mathcal{S}}|g(\boldsymbol{x})|$, we have

$$
\left|\int_{\mathcal{S}} g d \nu-I_{K} g\right| \leq\left(2 \rho L \varepsilon+\frac{2 \delta-(4+3 \delta) \varepsilon^{2}}{\delta-(4+3 \delta) \varepsilon^{2}}\|g\|_{\infty}\right) A(\mathcal{S}) .
$$

Remark 3. Results like this theorem have been proposed in Theorem 2.5 in [22]. We put the proof of this theorem into the Appendix of the paper.

Let

$$
V=\bigcup_{\triangle \in \mathcal{T}_{K}} V(\triangle)
$$


and

$$
K_{r}(\mathbf{x})=\bigcup_{\mathbf{y} \in V_{r}(\mathbf{x})} R(\mathbf{y})
$$

where $V_{r}(\mathbf{x})=\left\{\mathbf{y} \in V: d_{\mathcal{S}}(\mathbf{x}, \mathbf{y}) \leq r\right\}$, and $R(\mathbf{x})$ denotes the union of the triangles sharing the vertex $\mathrm{x} \in V$, i.e., one ring neighbourhood of the vertex $\mathbf{x}$. For any $\mathbf{x} \in V$, by constructing the discretized version of the integral (7), we obtain the discretization of LBO, denoted as $\mathrm{L}_{K_{r}}^{t}$ :

$$
\begin{aligned}
\mathrm{L}_{K_{r}}^{t} f(\mathbf{x}) & =\frac{1}{4 \pi t^{2}} \sum_{\triangle \in K_{r}(\mathbf{x})} \frac{A(\triangle)}{3} \sum_{\mathbf{p} \in V(\triangle)} e^{-\frac{d_{\mathcal{S}}(\mathbf{p}, \mathbf{x})^{2}}{4 t}}(f(\mathbf{p})-f(\mathbf{x})) \\
& =\frac{1}{4 \pi t^{2}} \sum_{\mathbf{p} \in V_{r}(\mathbf{x})} \frac{A(\mathbf{p})}{3} e^{-\frac{d_{\mathcal{S}}(\mathbf{p}, \mathbf{x})^{2}}{4 t}}(f(\mathbf{p})-f(\mathbf{x})),
\end{aligned}
$$

where $A(\mathbf{p})$ denotes the area of the one ring neighbourhood of the vertex $\mathbf{p}$, the parameters $t$ and $r$ are functions of the point $\mathbf{x}$, which will allow the algorithm to adapt to the local mesh size. We will discuss how to calculate the parameters adaptively in detail in Section 5 .

The following theorem shows the quality of approximating $\mathrm{F}_{\mathcal{S}_{r}}^{t}$ by $\mathrm{L}_{K_{r}}^{t}$ in terms of the parameters of the surface and the triangular mesh.

Theorem 9. Let $K_{\varepsilon, \delta}$ be an approximating triangular mesh of $\mathcal{S}$ with $\varepsilon<1 / 10$ and $\delta \geq 4 / 37$. Given a function $f \in C^{1}(\mathcal{S})$, let $\|f\|_{\infty}=\sup _{x \in \mathcal{S}}|f(\boldsymbol{x})|$ and $\left\|\nabla_{\mathcal{S}} f\right\|_{\infty}=\sup _{x \in \mathcal{S}}\left\|\nabla_{\mathcal{S}} f\right\|$, for any point $\boldsymbol{x} \in \mathcal{S}$, we have

$$
\begin{aligned}
\left|L_{K_{r}}^{t} f(\boldsymbol{x})-F_{\mathcal{S}_{r}}^{t} f(\boldsymbol{x})\right| \leq & \frac{A(\mathcal{S})}{2 \pi t^{2}} \cdot\left\{\left[C \rho \varepsilon t^{-\frac{1}{2}}+\frac{2 \delta-(4+3 \delta) \varepsilon^{2}}{\delta-(4+3 \delta) \varepsilon^{2}}\right]\|f\|_{\infty}\right. \\
& \left.+\rho \varepsilon\left\|\nabla_{\mathcal{S}} f\right\|_{\infty}\right\} .
\end{aligned}
$$

Proof. First, set $g(\mathbf{y})=\frac{1}{4 \pi t^{2}} e^{-\frac{d_{\mathcal{S}}(\mathbf{x}, \mathbf{y})^{2}}{4 t}}(f(\mathbf{y})-f(\mathbf{x}))$. Comparing Eqn 77 and Eqn (14), we obtain that

$$
\left|\mathrm{L}_{K_{r}}^{t} f(\mathbf{x})-\mathrm{F}_{\mathcal{S}_{r}}^{t} f(\mathbf{x})\right|=\left|\int_{\mathcal{S}_{r}} g d \nu-\mathrm{I}_{K_{r}} g\right| .
$$

By Theorem 8 to bound the above quantity, we bound $\|g\|_{\infty}$ and the Lipschitz constant of $g$. It is easy to verify that

$$
\|g\|_{\infty} \leq \frac{1}{2 \pi t^{2}}\|f\|_{\infty} .
$$


For $\operatorname{Lip}(g)$, since $g$ is $C^{1}$-continuous, it is upper bounded by

$$
\left\|\nabla_{\mathcal{S}} g\right\|_{\infty}=\sup _{\mathbf{y} \in \mathcal{S}}\left\|\nabla_{\mathcal{S}} g(\mathbf{y})\right\| .
$$

On the other hand, we have

$$
\left\|\nabla_{\mathcal{S}} g(\mathbf{y})\right\| \leq \frac{1}{4 \pi t^{2}}\left(2\|f\|_{\infty} \cdot\left\|\nabla_{\mathcal{S}} e^{-\frac{d_{\mathcal{S}}(\mathbf{x}, \mathbf{y})^{2}}{4 t}}\right\|+\left\|\nabla_{\mathcal{S}} f(\mathbf{y})\right\|\right) .
$$

To bound $\left\|\nabla_{\mathcal{S}} e^{-\frac{d_{\mathcal{S}}(\mathbf{x}, \mathbf{y})^{2}}{4 t}}\right\|$, let $\mathbf{y}\left(u^{1}, u^{2}\right)$ be the parametric representation of $\mathcal{S}$, then we have $\mathbf{y}_{u^{\alpha}}=\frac{\partial \mathbf{y}}{\partial u^{\alpha}}, g_{\alpha \beta}=\left\langle\mathbf{y}_{u^{\alpha}}, \mathbf{y}_{u^{\beta}}\right\rangle,\left[g^{\alpha \beta}\right]=\left[g_{\alpha \beta}\right]^{-1}, \varphi(\mathbf{y})=$ $\varphi\left(\mathbf{y}\left(u^{1}, u^{2}\right)\right)=e^{-\frac{d_{\mathcal{S}}(\mathbf{x}, \mathbf{y})^{2}}{4 t}}$ and $\varphi_{u^{\alpha}}=\frac{\partial \varphi}{\partial u^{\alpha}}$. By definition,

$$
\nabla_{\mathcal{S}} \varphi=\left[\mathbf{y}_{u^{1}}, \mathbf{y}_{u^{2}}\right]\left[g^{\alpha \beta}\right]\left[\varphi_{u^{1}}, \varphi_{u^{2}}\right]^{\mathrm{T}}
$$

then we have

$$
\begin{aligned}
\left\|\nabla_{\mathcal{S}} \varphi\right\|^{2} & =\left[\varphi_{u^{1}}, \varphi_{u^{2}}\right]\left[g^{\alpha \beta}\right]\left[\mathbf{y}_{u^{1}}, \mathbf{y}_{u^{2}}\right]^{\mathrm{T}}\left[\mathbf{y}_{u^{1}}, \mathbf{y}_{u^{2}}\right]\left[g^{\alpha \beta}\right]\left[\varphi_{u^{1}}, \varphi_{u^{2}}\right]^{\mathrm{T}} \\
& =\left[\varphi_{u^{1}}, \varphi_{u^{2}}\right]\left[g^{\alpha \beta}\right]\left[\varphi_{u^{1}}, \varphi_{u^{2}}\right]^{\mathrm{T}} .
\end{aligned}
$$

Since $\left[g_{\alpha \beta}\right]$ is fully dependent on the surface, $\left[g^{\alpha \beta}\right]$ is bounded for smooth surface. Hence, we have

$$
\left\|\nabla_{\mathcal{S}} \varphi\right\|^{2} \leq C\left(\left|\varphi_{u^{1}}\right|^{2}+\left|\varphi_{u^{2}}\right|^{2}+\left|\varphi_{u^{1}} \varphi_{u^{2}}\right|\right)
$$

where $C$ refers to a certain positive constant in this paper. To bound $\left\|\nabla_{\mathcal{S}} \varphi\right\|^{2}$, we need to bound $\left|\varphi_{u^{1}}\right|$ and $\left|\varphi_{u^{2}}\right|$. For $\left|\varphi_{u^{1}}\right|$, we have

$$
\begin{aligned}
\left|\varphi_{u^{1}}\right| & =e^{-\frac{d_{\mathcal{S}}(\mathbf{x}, \mathbf{y})^{2}}{4 t}} \frac{d_{\mathcal{S}}(\mathbf{x}, \mathbf{y})}{2 t}\left|\frac{\partial d_{\mathcal{S}}(\mathbf{x}, \mathbf{y})}{\partial u^{1}}\right| \\
& \leq C e^{-\frac{d_{\mathcal{S}}(\mathbf{x}, \mathbf{y})^{2}}{4 t}} \frac{d_{\mathcal{S}}(\mathbf{x}, \mathbf{y})}{2 t} \leq C \frac{1}{\sqrt{t}}
\end{aligned}
$$

where $\left|\frac{\partial d_{\mathcal{S}}(\mathbf{x}, \mathbf{y})}{\partial u^{1}}\right|$ is bounded by some positive constant $C$ for a smooth surface $\mathcal{S}$ and the last inequality holds as $z / e^{z^{2}} \leq 1$ for any real number $z$. Similarly, we can obtain $\left|\varphi_{u^{2}}\right| \leq C \frac{1}{\sqrt{t}}$. It then follows that

$$
\left\|\nabla_{\mathcal{S}} \varphi\right\|^{2} \leq C \frac{1}{t}
$$

and

$$
\left\|\nabla_{\mathcal{S}} e^{-\frac{d_{\mathcal{S}}(\mathbf{x}, \mathbf{y})^{2}}{4 t}}\right\|=\left\|\nabla_{\mathcal{S}} \varphi\right\| \leq C \frac{1}{\sqrt{t}}
$$


Thus,

$$
\left\|\nabla_{\mathcal{S}} g\right\|_{\infty}=\sup _{\mathbf{y} \in \mathcal{S}}\left\|\nabla_{\mathcal{S}} g(\mathbf{y})\right\| \leq \frac{1}{4 \pi t^{2}}\left(\frac{C\|f\|_{\infty}}{\sqrt{t}}+\left\|\nabla_{\mathcal{S}} f\right\|_{\infty}\right) .
$$

Combining with Theorem 8 and putting everything together, we prove the theorem.

Theorem 10. Let $K_{\varepsilon, \delta}$ be an approximating triangular mesh of $\mathcal{S}$, we $\operatorname{set} t(\varepsilon)=$ $\varepsilon^{\alpha}$ for an arbitrary fixed positive number $0<\alpha<2 / 5$. Then for any $f \in C^{2}(\mathcal{S})$, we have

$$
\lim _{\varepsilon \rightarrow 0} \sup _{K_{\varepsilon, \delta}}\left\|L_{K_{r}}^{t(\varepsilon)} f-\Delta_{\mathcal{S}} f\right\|_{\infty}=0
$$

where the supremum is taken over all approximating triangular meshes $K_{\varepsilon, \delta}$ of $\mathcal{S}$.

Remark 4. Results like this theorem have been proposed in Theorem 2.1 in 22. We put the proof of this theorem into the Appendix of the paper.

By now, we have proven that, as the approximating mesh of the surface becomes denser, our discretization of LBO on $K$ converges to the LaplaceBeltrami operator on $\mathcal{S}$.

\section{Numerical Experiments}

This section aims to exhibit the numerical behaviours of our discretization of LBO defined by (14. To show the numerical convergence of $\mathrm{L}_{K_{r}}^{t}$, we compute the mean curvature using (4). Let $d$ denote the maximal edge length of the triangular mesh $K$, which is called the mesh size of $K$.

Since our algorithm is based on a no-boundary condition and it holds for interior points of a surface, we consider six two-variable functions over the region 
$[-3,3] \times[-3,3]$ in the $u v$-plane as surfaces in $\mathbf{R}^{3}$,

$$
\begin{aligned}
& F_{1}(u, v)=\sqrt{18-u^{2}-v^{2}} \\
& F_{2}(u, v)=u^{2}+v^{2} \\
& F_{3}(u, v)=\tanh (9 v-9 u) \\
& F_{4}(u, v)=\frac{1.25+\cos (5.4 v)}{6+6(3 u-1)^{2}} \\
& F_{5}(u, v)=\exp \left(-\frac{81}{16}\left(u^{2}+v^{2}\right)\right) \\
& F_{6}(u, v)=\sin (5 u) \sin (5 v)
\end{aligned}
$$

Their exact mean curvatures can be easily computed analytically. The surfaces are triangulated by triangulating the planar domain first and then mapping the planar triangulation onto the surface via the selected bivariate functions. Both the exact and approximated mean curvatures are computed at some selected domain points $\left(u_{i}, v_{j}\right) \in[-1,1] \times[-1,1]$. As the first test case, the planar domain is triangulated as shown in Figure 2(a), and the points are chosen as $\left(u_{i}, v_{j}\right)=\left(\frac{i}{5}, \frac{j}{5}\right)$ for $i=-5, \ldots,-1,0,1, \ldots, 5 ; j=-5, \ldots,-1,0,1, \ldots, 5$. The second test case is an unstructured domain triangulation as shown in Figure 2(b), and we compute the exact and approximated mean curvatures at all the vertices in $[-1,1] \times[-1,1]$.

In the numerical experiments, we need to choose the input values for parameters $t$ and $r$ in (14). In order to compute $t$ adaptively, we adopt a fitting method. Firstly, we choose the domain as $[0,1] \times[0,1]$ and set $r=\frac{\sqrt{2}}{2}$, then for the regular mesh, the fitting method is as follows:

(1) Triangulate the domain around the point $(0.5,0.5)$ locally as shown in Figure 2(a) with $d_{i}=\frac{1}{20 \cdot 2^{i}}$, where $i=0,1,2,3$.

(2) For the point $(0.5,0.5)$ in each mesh $K_{i}$, set a series of different values to $t$, for example, $t_{j}=j * 0.0001, j=1,2, \cdots, 1000$, compute the absolute errors between the exact and approximated mean curvatures of the surfaces defined by the functions

$$
\left\{B_{k}(u, v)\right\}_{k=0}^{2}=\left\{u^{2},(u+1)(v+1), v^{2}\right\}
$$




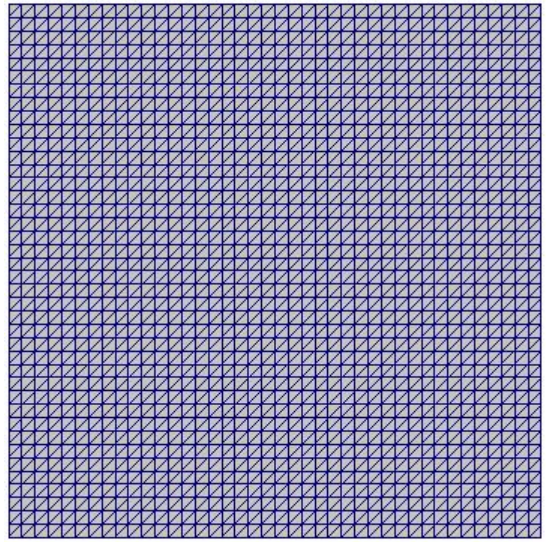

(a)

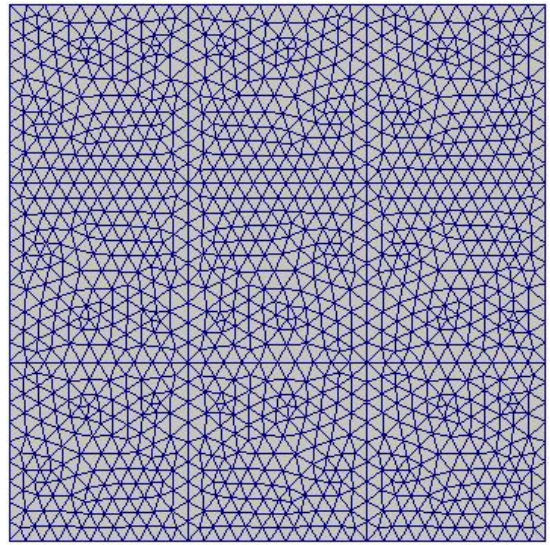

(b)

Figure 2: Triangulation of the domain. (a) Three directional triangular partition; (b) Unstructured triangular partition.

respectively. Choose $\left(d_{i}, t_{i, k}\right), k=0,1,2$, with the smallest absolute error.

(3) Let $t=\beta d^{\gamma}$, and determine the coefficients $\beta, \gamma>0$ such that

$$
\beta d_{i}^{\gamma}=t_{i, k}, \quad i=0,1,2,3 ; \quad k=0,1,2
$$

hold in the least square sense. By taking the natural logarithm on both sides of these equations, we obtain

$$
\ln \beta+\gamma \ln d_{i}=\ln t_{i, k}, \quad i=0,1,2,3 ; \quad k=0,1,2 .
$$

This system is solved by solving its normal equation. The fitting result is shown in Figure 4 (a) and

$$
t=0.2601 d^{1.7947}
$$

where $d$ denotes the local mesh size around the point $\mathbf{x}$, that is, the average distance between $\mathbf{x}$ and its one ring neighbor vertices.

Similarly for the unstructured mesh, our fitting method is as follows:

(1) The original triangular partition of the domain $[0,1] \times[0,1]$ is shown in Figure 3 It is recursively subdivided by linear bisection for three times. 


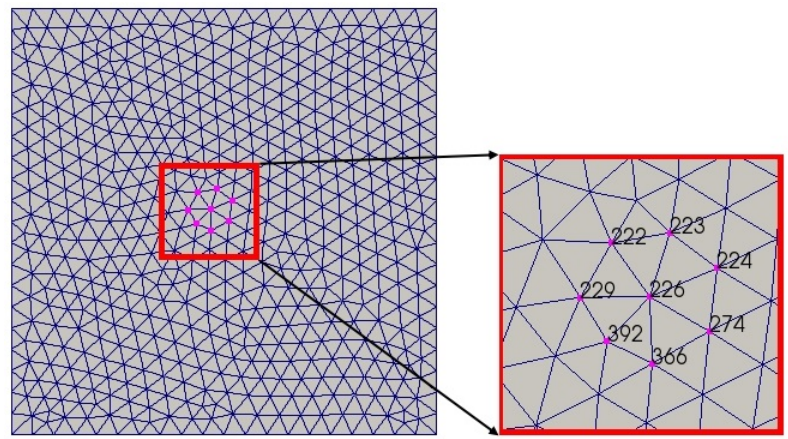

Figure 3: Unstructured mesh for fitting.

For the vertex 226 , it is easy to see that its valance is 7 , one of its one ring neighbour vertices, whose index is 392 , has a valance of 5 , and others are all valance- 6 vertices.

(2) For the vertex 226 and its one ring neighbour vertices in each mesh $K_{i}$, compute the local mesh sizes respectively, then average them to obtain $d_{i}$ for each mesh.

(3) For the vertex 226 and its one ring neighbour vertices in each mesh $K_{i}$, set a series of different values to $t$, compute the absolute errors between the exact and approximated mean curvatures of the surfaces defined by $(19)$ at these vertices respectively, then average them to obtain the averaged absolute error at vertex 226. Choose $\left(d_{i}, t_{i, k}\right)$ with the smallest absolute error.

(4) Let $t=\beta d^{\gamma}$, and determine the coefficients $\beta, \gamma>0$ such that

$$
\beta d_{i}^{\gamma}=t_{i, k}, \quad i=0,1,2,3 ; \quad k=0,1,2
$$

hold in the least square sense. By taking the natural logarithm on both sides of these equations, we obtain

$$
\ln \beta+\gamma \ln d_{i}=\ln t_{i, k}, \quad i=0,1,2,3 ; \quad k=0,1,2 .
$$

Again, this system is solved by solving its normal equation. The fitting result is shown in Figure 4(b) and

$$
t=0.0568 d^{0.9215} .
$$




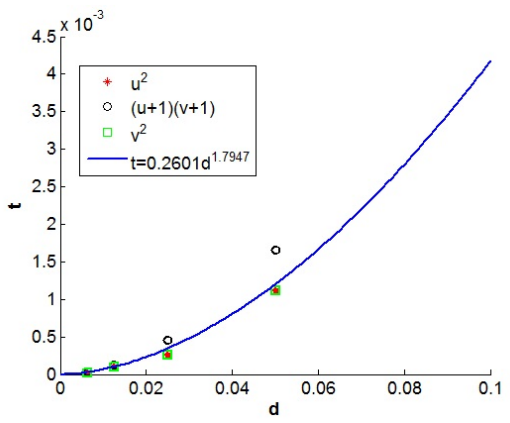

(a)

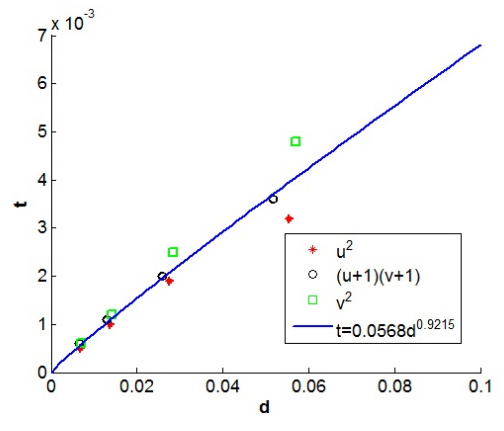

(b)

Figure 4: The choice of the parameter $t$ according to the local mesh size $d$ for the regular mesh (a) and unstructured mesh (b) respectively.

For the parameter $r$, from Eqn $(9)$ with the precomputed $t$, we have

$$
\begin{aligned}
\left|\mathrm{F}_{\mathcal{S}_{r}}^{t} \mathbf{x}(p)-\mathrm{F}_{\mathcal{S}}^{t} \mathbf{x}(\omega)\right| \leq & \frac{1}{4 \pi t^{2}} e^{-\frac{r^{2}}{4 t}}\left(\left(|x(\omega)| A(\mathcal{S})+\|x\|_{L_{1}}\right)^{2}\right. \\
& +\left(|y(\omega)| A(\mathcal{S})+\|y\|_{L_{1}}\right)^{2} \\
& \left.+\left(|z(\omega)| A(\mathcal{S})+\|z\|_{L_{1}}\right)^{2}\right)^{\frac{1}{2}} \leq \eta
\end{aligned}
$$

where $\mathbf{x}(\omega)=[x(\omega), y(\omega), z(\omega)]^{\mathrm{T}}$ is a parametric representation of $\mathcal{S}$, and $\eta>0$ is the threshold. Set

$$
\begin{aligned}
T= & \left(\left(|x(\omega)| A(\mathcal{S})+\|x\|_{L_{1}}\right)^{2}+\left(|y(\omega)| A(\mathcal{S})+\|y\|_{L_{1}}\right)^{2}\right. \\
& \left.+\left(|z(\omega)| A(\mathcal{S})+\|z\|_{L_{1}}\right)^{2}\right)^{\frac{1}{2}}
\end{aligned}
$$

then we obtain

$$
r \geq\left(-4 h \ln \frac{4 \pi t^{2} \eta}{T}\right)^{\frac{1}{2}}
$$

In our numerical experiments, given $\eta>0$, the precomputed $t$ and $T$, we just take $r$ as

$$
r=\left(-4 h \ln \frac{4 \pi t^{2} \eta}{T}\right)^{\frac{1}{2}}
$$

To observe the convergent property, finer and finer domain triangulations are generated. For case (a) in Figure 2 , $d$ is taken to be $\frac{1}{10}, \frac{1}{20}, \frac{1}{40}, \frac{1}{80}$ or $\frac{1}{160}$. For case (b), the domain is recursively subdivided by the bisection linear 
subdivision. Hence, $d=d_{0}, \frac{d_{0}}{2}, \frac{d_{0}}{4}, \frac{d_{0}}{8}$ or $\frac{d_{0}}{16}$, where $d_{0}=0.327804$ is the maximal edge length of the triangulation as shown in Figure 2(b).

To measure the error between our discretization of the LBO and the surface Laplacian, we consider the maximal error between the approximated mean curvature computed by our proposed approximate operator $\mathrm{L}_{K_{r}}^{t}$ and the exact mean curvature computed from the continuous surfaces defined by $F_{i}$. The results are shown in Tables 1 and 2 for the regular and unstructured meshes respectively. It is easy to see that the approximation errors of all six surfaces by our method always approach to zero as $d \rightarrow 0$, then we come to the conclusion that our proposed approximate operator $\mathrm{L}_{K_{r}}^{t}$ converges to the surface Laplacian under the $L_{\infty}$ norm.

Figures 5 and 6 show the distributions of the absolute errors between the exact mean curvatures and the approximated mean curvatures, which are computed by our method on surfaces defined by 18 with regular and unstructured meshes respectively. We can observe that our method achieves pretty good approximations in flat regions, but the absolute errors are relatively large at sharp edges or spikes. This makes sense since the approximated geodesic distance between any two points on triangular mesh $K$ is closely related to the geodesic distance on the original surface $\mathcal{S}$ in gently changed regions, while it may result in relatively larger error in sharp regions.

To better analyse the convergence property of our discretization of the LBO, we compare the behaviours of our method with those of the cotangent scheme [15], mesh Laplacian [22] and PCD Laplacian 23] as the mesh becomes denser and denser. Similar to the computation of our method, we also compute the maximal errors between the exact mean curvature computed from the continuous surfaces defined by (18) and the approximated mean curvatures computed by the above three methods for the regular and unstructured meshes shown in Figure 2, The numerical results are shown in Tables 1 and 2, We can observe that the maximal absolute errors of all six surfaces using our method always approach to zero as the mesh sizes tend to zero. In contrast, finer mesh does not lead to lower approximation errors by the cotangent scheme, mesh Lapla- 
Table 1: The maximal errors of four methods for regular meshes in Figure 2 a).

\begin{tabular}{|c|c|c|c|c|c|c|}
\hline & Mesh 1 & Mesh 2 & Mesh 3 & Mesh 4 & Mesh 5 \\
\hline & & $\left(441^{\mathrm{a}}, 800\right)^{\mathrm{p}}$ & $(1,681,3,200)$ & $(6,561,12,800)$ & $(25,921,51,200)$ & $(103,041,204,800)$ \\
\hline \multirow{4}{*}{$F_{1}(u, v)$} & Cotangent Scheme & 0.235702 & 0.235702 & 0.235702 & 0.235702 & 0.235702 \\
\hline & Mesh Laplacian & 0.000411 & 0.000468 & 0.000430 & 0.000416 & 0.000412 \\
\hline & PCD Laplacian & 0.001552 & 0.003481 & 0.008454 & 0.004977 & 0.000901 \\
\hline & Our Discretization & 0.000060 & 0.000020 & 0.000006 & 0.000002 & 0.000001 \\
\hline \multirow{4}{*}{$F_{2}(u, v)$} & Cotangent Scheme & 1.508710 & 1.524443 & 1.528437 & 1.529439 & 1.529690 \\
\hline & Mesh Laplacian & 0.137564 & 0.147730 & 0.150068 & 0.150726 & 0.150856 \\
\hline & PCD Laplacian & 0.128634 & 0.043275 & 0.010182 & 0.010499 & 0.004664 \\
\hline & Our Discretization & 0.106606 & 0.034988 & 0.010797 & 0.003247 & 0.000966 \\
\hline \multirow{4}{*}{$F_{3}(u, v)$} & Cotangent Scheme & 0.803238 & 2.623072 & 3.268838 & 3.446476 & 3.491986 \\
\hline & Mesh Laplacian & 1.129171 & 1.075224 & 1.155793 & 1.166884 & 1.169671 \\
\hline & PCD Laplacian & 1.739820 & 0.791107 & 0.201265 & 0.263039 & 0.299344 \\
\hline & Our Discretization & 0.817050 & 0.267496 & 0.070618 & 0.019475 & 0.005532 \\
\hline \multirow{4}{*}{$F_{4}(u, v)$} & Cotangent Scheme & 2.396574 & 2.519787 & 2.554907 & 2.564053 & 2.566364 \\
\hline & Mesh Laplacian & 1.219628 & 1.192671 & 1.185124 & 1.183230 & 1.182732 \\
\hline & PCD Laplacian & 0.848218 & 0.241403 & 0.058406 & 0.024557 & 0.015631 \\
\hline & Our Discretization & 0.945236 & 0.290051 & 0.071237 & 0.018535 & 0.005268 \\
\hline \multirow{4}{*}{$F_{5}(u, v)$} & Cotangent Scheme & 1.319973 & 2.020372 & 3.005013 & 3.280313 & 3.351187 \\
\hline & Mesh Laplacian & 5.854427 & 5.848452 & 5.847980 & 5.848257 & 5.848202 \\
\hline & PCD Laplacian & 8.480840 & 2.580990 & 0.690788 & 0.185750 & 0.083480 \\
\hline & Our Discretization & 5.284975 & 2.730238 & 1.152661 & 0.406840 & 0.128737 \\
\hline \multirow{4}{*}{$F_{6}(u, v)$} & Cotangent Scheme & 4.168940 & 4.634181 & 6.063968 & 6.596706 & 6.744826 \\
\hline & Mesh Laplacian & 4.185271 & 3.726886 & 3.770037 & 3.780960 & 3.783662 \\
\hline & PCD Laplacian & 5.389650 & 5.944800 & 6.644630 & 1.852490 & 0.580877 \\
\hline & Our Discretization & 4.084806 & 3.381458 & 2.162143 & 1.054748 & 0.365619 \\
\hline
\end{tabular}

a the number of points in a triangular mesh

$\mathrm{b}$ the number of triangles in a triangular mesh

cian and PCD Laplacian. We can see that our discretization provides better approximation than the mesh Laplacian and cotangent scheme, and the PCD Laplacian also provides better approximation except the unstructured mesh defined by $F_{3}$ which has a waterfall shape. The reason why the PCD Laplacian works badly for these meshes is that this method projects the neighbour dataset of the point of interest onto its tangent plane, which introduces significant errors. It is easy to see that the PCD Laplacian achieves better approximation than our discretization on the surfaces defined by $F_{2}$ and $F_{5}$ which have single peaks, when the point cloud is dense enough. 
Table 2: The maximal errors of four methods for unstructured meshes in Figure 2 b).

\begin{tabular}{|c|c|c|c|c|c|c|}
\hline & Mesh 1 & Mesh 2 & Mesh 3 & Mesh 4 & Mesh 5 \\
\hline & & $(152,263)$ & $(566,1,052)$ & $(2,183,4,208)$ & $(8,573,16,832)$ & $(33,977,67,328)$ \\
\hline \multirow{4}{*}{$F_{1}(u, v)$} & Cotangent Scheme & 0.235712 & 0.235705 & 0.235703 & 0.235702 & 0.235702 \\
\hline & Mesh Laplacian & 0.216080 & 0.014542 & 0.000771 & 0.000421 & 0.000322 \\
\hline & PCD Laplacian & 0.001683 & 0.005028 & 0.013758 & 0.095672 & 0.287526 \\
\hline & Our Discretization & 0.101205 & 0.032905 & 0.013538 & 0.011564 & 0.006840 \\
\hline \multirow{4}{*}{$F_{2}(u, v)$} & Cotangent Scheme & 1.814936 & 1.944597 & 1.977632 & 1.985597 & 1.987404 \\
\hline & Mesh Laplacian & 1.541950 & 0.142515 & 0.145452 & 0.146267 & 0.146461 \\
\hline & PCD Laplacian & 1.498230 & 0.138436 & 0.052086 & 0.013285 & 0.029403 \\
\hline & Our Discretization & 0.240210 & 0.162081 & 0.104578 & 0.058352 & 0.031748 \\
\hline \multirow{4}{*}{$F_{3}(u, v)$} & Cotangent Scheme & 3.852991 & 2.893134 & 3.830829 & 4.602100 & 4.840330 \\
\hline & Mesh Laplacian & 6.835887 & 3.377893 & 2.206625 & 2.286917 & 2.311476 \\
\hline & PCD Laplacian & 3.324200 & 2.358150 & 4.457640 & 6.918110 & 14.614000 \\
\hline & Our Discretization & 4.535904 & 1.942065 & 1.530049 & 1.244348 & 0.898454 \\
\hline \multirow{4}{*}{$F_{4}(u, v)$} & Cotangent Scheme & 2.796931 & 3.598438 & 3.691693 & 3.685935 & 3.674926 \\
\hline & Mesh Laplacian & 0.830878 & 0.768244 & 0.750365 & 0.745610 & 0.744430 \\
\hline & PCD Laplacian & 1.605960 & 0.496808 & 0.179935 & 0.323418 & 0.634843 \\
\hline & Our Discretization & 1.158992 & 0.745525 & 0.443030 & 0.232326 & 0.119999 \\
\hline \multirow{4}{*}{$F_{5}(u, v)$} & Cotangent Scheme & 1.691123 & 5.564102 & 7.846315 & 8.482160 & 8.628272 \\
\hline & Mesh Laplacian & 5.126129 & 4.517994 & 4.440401 & 4.422669 & 4.418278 \\
\hline & PCD Laplacian & 7.769850 & 6.531170 & 0.813982 & 0.396653 & 0.869729 \\
\hline & Our Discretization & 5.562846 & 4.468237 & 3.372483 & 2.446122 & 1.648161 \\
\hline \multirow{4}{*}{$F_{6}(u, v)$} & Cotangent Scheme & 17.358684 & 5.647897 & 12.910192 & 19.308704 & 21.243011 \\
\hline & Mesh Laplacian & 20.929625 & 17.808631 & 17.768711 & 17.774103 & 17.775265 \\
\hline & PCD Laplacian & 20.697100 & 20.475700 & 20.239800 & 17.591600 & 2.056850 \\
\hline & Our Discretization & 19.707274 & 17.760350 & 15.371045 & 13.074602 & 10.619516 \\
\hline
\end{tabular}

\section{Conclusion}

In this paper, we have developed an adaptive algorithm for approximating the Laplace-Beltrami operator on a triangular mesh with point-wise convergence guarantee. Such convergence is required in many applications, where quantities, such as mean curvature, need to be estimated at each node of the mesh. The convergence result does not require the aspect ratio of mesh elements to be bounded. Experimental results show that our algorithm indeed exhibits convergence empirically, and outperforms current popular methods in accuracy. Especially, our method provides quite good approximation of the LBO for surfaces with sharp features. In the future, we will investigate symmetrizable 


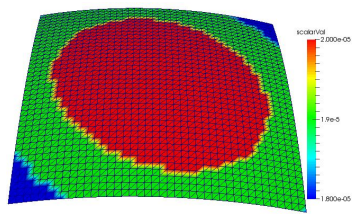

(a)

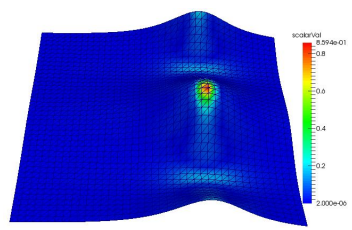

(d)

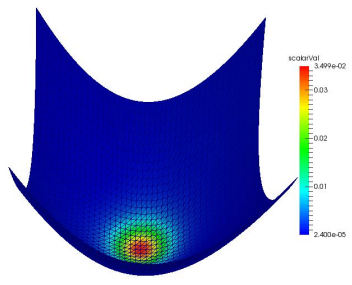

(b)

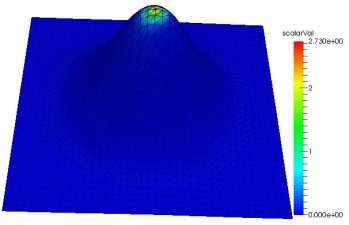

(e)

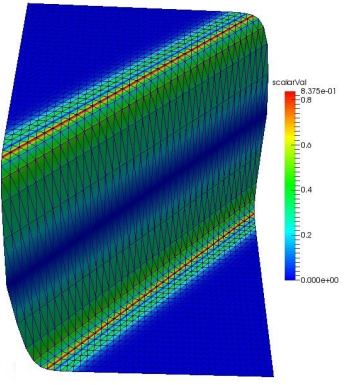

(c)

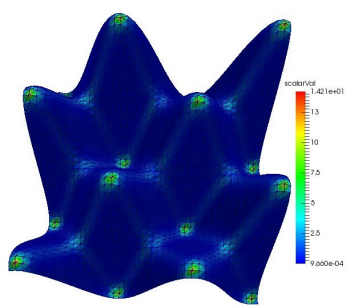

(f)

Figure 5: The distributions of the absolute error between the exact and approximated mean curvatures on six surfaces (a-f) defined by 18 with regular meshes.

discrete LBO based on our method and then build a set of orthogonal bases for spectral analysis over triangulated manifold surfaces.

Acknowledgment

X. Li and G. Xu were supported in part by NSFC under the Grants (11101401, 81173663), and NSFC Fund for Creative Research Groups of China (Grant No. 11321061). Y. Zhang was supported in part by PECASE Award N000141410234 and NSF CAREER Award OCI-1149591. The authors would also like to thank Mikhail Belkin (The Ohio State University) for the helpful inspiration of this work.

Appendix

Proof of Lemma 5] As shown in Figure 7] suppose the point $\mathbf{p}$ is contained in the triangle $\triangle \in \mathcal{T}_{K}$ and point $\mathbf{q}$ is any vertex of $\triangle$. Let $T_{\mathbf{q}}$ denote the tangent 


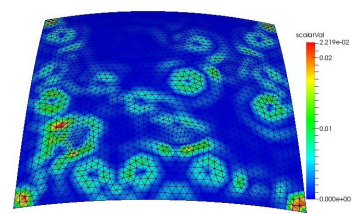

(a)

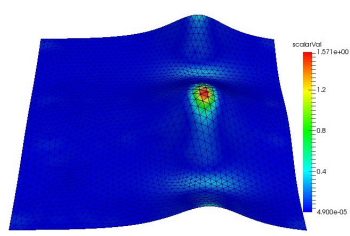

(d)

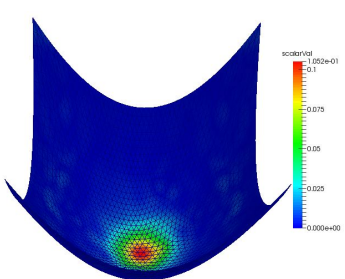

(b)

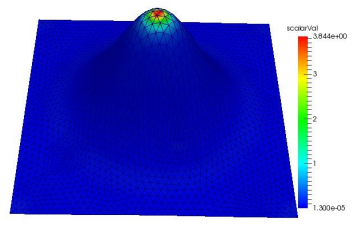

(e)

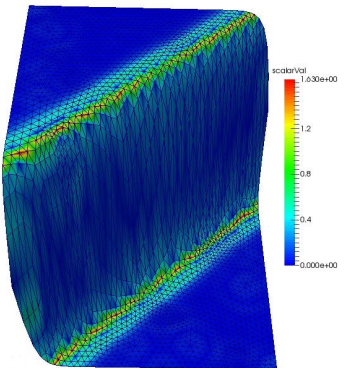

(c)

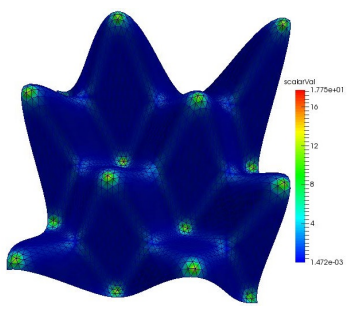

(f)

Figure 6: The distributions of the absolute error between the exact and approximated mean curvatures on six surfaces (a-f) defined by 18 with unstructured meshes.

plane at q. Assume that $B$ and $B^{\prime}$ are the two balls of radius $\rho$ tangentially touching $\mathcal{S}$ at $\mathbf{q}$ on each side of $T_{\mathbf{q}}$, and the centers of $B$ and $B^{\prime}$ are $\mathbf{o}$ and $\mathbf{o}^{\prime}$, respectively.

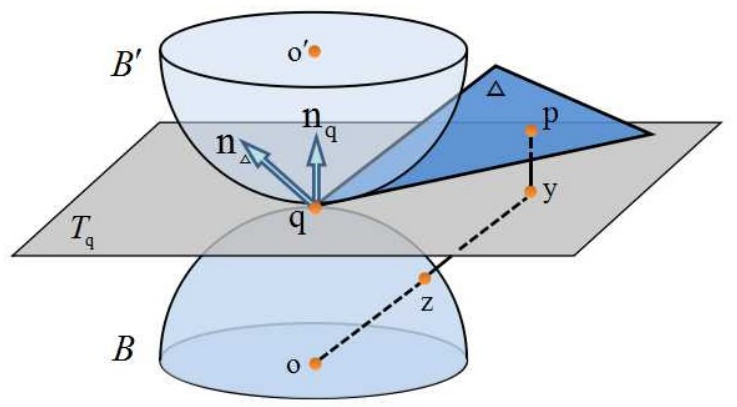

Figure 7: Illustration of Lemma 5 
Obviously, the angle between $T_{\mathbf{q}}$ and the plane passing through $\triangle$ is $\angle\left(\mathbf{n}_{\triangle}, \mathbf{n}_{\mathbf{q}}\right)$, which implies that the angle between $T_{\mathbf{q}}$ and the line passing through $\mathbf{p}$ and $\mathbf{q}$ is at most $\angle\left(\mathbf{n}_{\triangle}, \mathbf{n}_{\mathbf{q}}\right)$. Let $\mathbf{y}$ denote the projection of $\mathbf{p}$ on $T_{\mathbf{q}}$. We have

$$
\|\mathbf{y}-\mathbf{q}\| \leq\|\mathbf{p}-\mathbf{q}\| \leq \varepsilon \rho
$$

and

$$
\|\mathbf{y}-\mathbf{p}\| \leq\|\mathbf{q}-\mathbf{p}\| \sin \angle\left(\mathbf{n}_{\triangle}, \mathbf{n}_{\mathbf{q}}\right) \leq\left(\frac{4}{\delta}+2\right) \varepsilon^{2} \rho .
$$

The last inequality follows from 100 and (11). Furthermore, we observe that one of the segments $[\mathbf{o}, \mathbf{y}]$ and $\left[\mathbf{o}^{\prime}, \mathbf{y}\right]$ must intersect with $\mathcal{S}$. Without loss of generality, we assume $[\mathbf{o}, \mathbf{y}]$ intersects with $\mathcal{S}$. Since $B$ does not contain any point from $\mathcal{S}$ except $\mathbf{q}$, we have $d(\mathbf{y}, \mathcal{S}) \leq\|\mathbf{y}-\mathbf{z}\|$, where $\mathbf{z}$ is the intersection between $[\mathbf{o}, \mathbf{y}]$ and $B$. This implies that

$$
d(\mathbf{y}, \mathcal{S}) \leq \sqrt{\rho^{2}+\|\mathbf{y}-\mathbf{q}\|^{2}}-\rho \leq\left(\sqrt{1+\varepsilon^{2}}-1\right) \rho \leq \varepsilon^{2} \rho,
$$

then we have

$$
d(\mathbf{p}, \mathcal{S}) \leq\|\mathbf{p}-\mathbf{y}\|+d(\mathbf{y}, \mathcal{S}) \leq\left(\frac{4}{\delta}+2\right) \varepsilon^{2} \rho+\varepsilon^{2} \rho=\left(\frac{4}{\delta}+3\right) \varepsilon^{2} \rho,
$$

which proves part (i) of the lemma.

For part (ii), since the map $\xi: K \rightarrow \mathcal{S}$ is bijective and continuous, it is a homeomorphism and part (ii) holds following (i).

Proof of Theorem 8. Since $\xi$ is bijective and the set of surface mesh faces $\left\{\xi(\triangle), \triangle \in \mathcal{T}_{K}\right\}$ partitions the surface $\mathcal{S}$, we have

$$
\begin{aligned}
\int_{\mathcal{S}} g(\mathbf{x}) d \nu(\mathbf{x}) & =\sum_{\triangle \in \mathcal{T}_{K}} \int_{\xi(\triangle)} g(\mathbf{x}) d \nu(\mathbf{x}) \\
& =\sum_{\triangle \in \mathcal{T}_{K}} \int_{\triangle} g(\xi(u, v)) J(u, v) d u d v,
\end{aligned}
$$

where $(u, v)$ is any orthogonal coordinate system on the plane passing through 
the triangle $\triangle$. It then follows from Lemma 6 that

$$
\begin{aligned}
& \left|\int_{\mathcal{S}} g(\mathbf{x}) d \nu(\mathbf{x})-\sum_{\triangle \in \mathcal{T}_{K}} \int_{\triangle} g(\xi(u, v)) d u d v\right| \\
= & \left|\sum_{\Delta \in \mathcal{T}_{K}} \int_{\triangle} g(\xi(u, v))(J(u, v)-1) d u d v\right| \\
\leq & \sum_{\triangle \in \mathcal{T}_{K}} \int_{\triangle}|g(\xi(u, v))|\left(\frac{1}{1-\left(\frac{4}{\delta}+3\right) \varepsilon^{2}}+1\right) d u d v \\
\leq & \frac{2 \delta-(4+3 \delta) \varepsilon^{2}}{\delta-(4+3 \delta) \varepsilon^{2}} A(K)\|g\|_{\infty} .
\end{aligned}
$$

On the other hand, for any vertex $\mathbf{p}$ of $\triangle$ and any point $\mathbf{x}=(u, v) \in \triangle$, we have $\|\mathbf{p}-\mathbf{x}\| \leq \varepsilon \rho$ by definition of $K_{\varepsilon, \delta}$. It then follows from Lemma 5 (i) and the triangle inequality that

$$
\|\mathbf{p}-\xi(\mathbf{x})\| \leq\|\mathbf{p}-\mathbf{x}\|+\|\mathbf{x}-\xi(\mathbf{x})\| \leq\left(\varepsilon+\left(\frac{4}{\delta}+3\right) \varepsilon^{2}\right) \rho .
$$

Since $\varepsilon<1 / 10$ and $\delta \geq 4 / 37$, we have $d_{\mathcal{S}}(\mathbf{p}, \xi(\mathbf{x})) \leq 2 \varepsilon \rho$ by Lemma 7 . Therefore

$$
|g(\xi(\mathbf{x}))-g(\mathbf{p})| \leq 2 \rho L \varepsilon
$$

where $L=\operatorname{Lip}(g)$ is the Lipschitz constant of $g$. This implies

$$
\left|\int_{\triangle}(g(\xi(u, v))-g(\mathbf{p})) d u d v\right| \leq 2 \rho L \varepsilon A(\triangle) .
$$

Note that $\mathbf{p}$ is an arbitrary vertex of $\triangle$, we have

$$
\begin{aligned}
& \left|\int_{\triangle} g(\xi(u, v)) d u d v-\frac{A(\triangle)}{3} \sum_{\mathbf{p} \in V(\triangle)} g(\mathbf{p})\right| \\
= & \frac{1}{3}\left|3 \int_{\triangle} g(\xi(u, v)) d u d v-\sum_{\mathbf{p} \in V(\triangle)} g(\mathbf{p}) A(\triangle)\right| \\
\leq & \frac{1}{3} \sum_{\mathbf{p} \in V(\triangle)}\left|\int_{\triangle}(g(\xi(u, v))-g(\mathbf{p})) d u d v\right| \leq 2 \rho L \varepsilon A(\triangle) .
\end{aligned}
$$

Combining it with Eqn (23), we have

$$
\left|\int_{\mathcal{S}} g d \nu-\mathrm{I}_{K} g\right| \leq 2 \rho L \varepsilon A(K)+\frac{2 \delta-(4+3 \delta) \varepsilon^{2}}{\delta-(4+3 \delta) \varepsilon^{2}} A(K)\|g\|_{\infty} .
$$


From Lemma 6, we have

$$
A(K) \leq A(\mathcal{S})
$$

Thus the theorem is proved.

Proof of Theorem 10. For any point $\mathbf{x} \in \mathcal{S}$, we have

$$
\left|\mathrm{L}_{K_{r}}^{t(\varepsilon)} f(\mathbf{x})-\Delta_{\mathcal{S}} f(\mathbf{x})\right| \leq\left|\mathrm{L}_{K_{r}}^{t(\varepsilon)} f(\mathbf{x})-\mathrm{F}_{\mathcal{S}_{r}}^{t(\varepsilon)} f(\mathbf{x})\right|+\left|\mathrm{F}_{\mathcal{S}_{r}}^{t(\varepsilon)} f(\mathbf{x})-\Delta_{\mathcal{S}} f(\mathbf{x})\right| .
$$

By choosing $t(\varepsilon)=\varepsilon^{\alpha}$, where $0<\alpha<2 / 5$ is an arbitrary fixed positive number, it follows from Theorem 9 that

$$
\left|\mathrm{L}_{K_{r}}^{t(\varepsilon)} f(\mathbf{x})-\Delta_{\mathcal{S}} f(\mathbf{x})\right| \leq O\left(\varepsilon^{1-\frac{5}{2} \alpha}\right)+\left|\mathrm{F}_{\mathcal{S}_{r}}^{t(\varepsilon)} f(\mathbf{x})-\Delta_{\mathcal{S}} f(\mathbf{x})\right| .
$$

The above big-O notation is a linear combination of $A(\mathcal{S}), \rho(\mathcal{S}),\|f\|_{\infty}$ and $\left\|\nabla_{\mathcal{S}} f\right\|_{\infty}$. On the other hand, we can obtain

$$
\lim _{\varepsilon \rightarrow 0}\left|F_{\mathcal{S}_{r}}^{t(\varepsilon)} f(\mathbf{x})-\Delta_{\mathcal{S}} f(\mathbf{x})\right|=0
$$

by Theorem 1 . Hence by taking the limit, we have

$$
\lim _{\varepsilon \rightarrow 0} \mathrm{~L}_{K_{r}}^{t(\varepsilon)} f(\mathbf{x})=\Delta_{\mathcal{S}} f(\mathbf{x})
$$

which proves the theorem by noting that the surface $\mathcal{S}$ is compact.

\section{References}

[1] M. Bertalmio, G. Sapiro, L.-T. Cheng, S. Osher, A framework for solving surface partial differential equations for computer graphics applications, CAM Report 00-43, UCLA, Mathematics Department.

[2] R. Kimmel, R. Malladi, N. Sochen, Image processing via the Beltrami operator, in: Computer Vision-ACCV'98, Springer, 1997, pp. 574-581.

[3] G. Sapiro, Geometric Partial Differential Equations and Image Analysis, Cambridge University Press, 2006.

[4] J. Weickert, Anisotropic Diffusion in Image Processing, Vol. 1, Teubner Stuttgart, 1998. 
[5] C. L. Bajaj, G. Xu, Anisotropic diffusion of surfaces and functions on surfaces, ACM Transactions on Graphics (TOG) 22 (1) (2003) 4-32.

[6] U. Clarenz, U. Diewald, M. Rumpf, Anisotropic geometric diffusion in surface processing, in: Proceedings of the Conference on Visualization'00, IEEE Computer Society Press, 2000, pp. 397-405.

[7] M. Desbrun, M. Meyer, P. Schröder, A. H. Barr, Implicit fairing of irregular meshes using diffusion and curvature flow, in: Proceedings of the 26th Annual Conference on Computer Graphics and Interactive Techniques, ACM Press/Addison-Wesley Publishing Co., 1999, pp. 317-324.

[8] M. Meyer, M. Desbrun, P. Schröder, A. H. Barr, Discrete differentialgeometry operators for triangulated 2-manifolds, in: Visualization and Mathematics III, Springer, 2003, pp. 35-57.

[9] R. Schneider, L. Kobbelt, Generating fair meshes with $G^{1}$ boundary conditions, in: Geometric Modeling and Processing 2000. Theory and Applications. Proceedings, IEEE, 2000, pp. 251-261.

[10] R. Schneider, L. Kobbelt, Geometric fairing of irregular meshes for freeform surface design, Computer Aided Geometric Design 18 (4) (2001) 359379.

[11] U. F. Mayer, Numerical solutions for the surface diffusion flow in three space dimensions, Computational and Applied Mathematics 20 (3) (2001) 361-379.

[12] B. M. ter Haar Romeny, Geometry-driven Diffusion in Computer Vision, Kluwer Academic Norwell, MA, 1994.

[13] U. Pinkall, K. Polthier, Computing discrete minimal surfaces and their conjugates, Experimental Mathematics 2 (1) (1993) 15-36.

[14] G. Taubin, A signal processing approach to fair surface design, in: Proceedings of the 22nd Annual Conference on Computer Graphics and Interactive Techniques, ACM, 1995, pp. 351-358. 
[15] G. Xu, Discrete Laplace-Beltrami operators and their convergence, Computer Aided Geometric Design 21 (8) (2004) 767-784.

[16] L. Demanet, Painless, highly accurate discretizations of the Laplacian on a smooth manifold, Technical report, Stanford, December 2006.

URL http://math.mit.edu/icg/papers/PainlessLB.pdf

[17] M. Wardetzky, Convergence of the cotangent formula: An overview, in: Discrete Differential Geometry, Vol. 38 of Oberwolfach Seminars, Birkhäuser Basel, 2008, pp. 275-286.

[18] D. Liu, G. Xu, Q. Zhang, A discrete scheme of Laplace-Beltrami operator and its convergence over quadrilateral meshes, Computers \& Mathematics with Applications 55 (6) (2008) 1081 - 1093.

[19] Y. Xiong, G. Li, G. Han, Mean Laplace-Beltrami operator for quadrilateral meshes, in: Transactions on Edutainment V, Vol. 6530 of Lecture Notes in Computer Science, Springer Berlin Heidelberg, 2011, pp. 189-201.

[20] M. Alexa, M. Wardetzky, Discrete Laplacians on general polygonal meshes, ACM Trans. Graph. 30 (4) (2011) 102:1-102:10.

[21] W. Carl, A Laplace operator on semi-discrete surfaces, Foundations of Computational Mathematics (2015) 1-36.

[22] M. Belkin, J. Sun, Y. Wang, Discrete Laplace operator on meshed surfaces, in: Proceedings of the Twenty-fourth Annual Symposium on Computational Geometry, ACM, 2008, pp. 278-287.

[23] M. Belkin, J. Sun, W. Yusu, Constructing Laplace operator from point clouds in $R^{d}$ (2009).

[24] J. Sun, M. Ovsjanikov, L. Guibas, A concise and provably informative multi-scale signature based on heat diffusion, Computer Graphics Forum 28 (5) (2009) 1383-1392. 
[25] Y. Liu, B. Prabhakaran, X. Guo, Point-based manifold harmonics, IEEE Transactions on Visualization and Computer Graphics 18 (10) (2012) 16931703.

[26] K. Hildebrandt, K. Polthier, On approximation of the Laplace-Beltrami operator and the Willmore energy of surfaces, Computer Graphics Forum 30 (5) (2011) 1513-1520.

[27] T. J. Willmore, Riemannian Geometry, Vol. 33, Clarendon Press Oxford, 1993.

[28] H. Federer, Curvature measures, Transactions of the American Mathematical Society (1959) 418-491.

[29] J.-M. Morvan, B. Thibert, Approximation of the normal vector field and the area of a smooth surface, Discrete \& Computational Geometry 32 (3) (2004) 383-400.

[30] J.-M. Morvan, B. Thibert, On the approximation of a smooth surface with a triangulated mesh, Computational Geometry 23 (3) (2002) 337-352. 